Check for updates

1 Queen's University Belfast

2 DATA-CAN

3 The European Cancer Organisation, Brussels, Belgium

4 Amsterdam UMC, Amsterdam, the Netherlands

Cite this as: BMJ 2022;376:0282 http://dx.doi.org/10.1136/bmj.o282

Published: 03 February 2022

\title{
Data must underpin our response to the covid-19 pandemic's disastrous impact on cancer
}

\author{
Unless we take action, the current pandemic will precipitate a future cancer epidemic, write Mark \\ Lawler and Mirjam Crul. Data driven approaches offer the best way forward
}

Mark Lawler, 1,2,3 Mirjam Crul ${ }^{4,3}$

One consequence of the global response to the covid-19 pandemic has been the disruption it has caused to health services and for patients with non-communicable diseases. We have previously highlighted, along with other commentators, the particularly devastating impact of the pandemic on people with cancer, causing significant strain across the entire cancer pathway, and leading to presentational, diagnostic, and treatment delays. ${ }^{1-4}$ This led one of us to warn in May 2021 that "cancer must not become the forgotten ' $\mathrm{C}$ ' in the fight against covid." 5

Nine months later, where do we stand? It has become apparent that the best way to tackle cancer care during the covid-19 pandemic is to define the challenges that European cancer health services and cancer patients face, and deploy this knowledge to deliver action for European cancer patients.

As early as April 2020, data from the Netherlands highlighted that there had been fewer cancer diagnoses between 24 February 2020 and 12 April 2020 compared with the period before the covid outbreak. ${ }^{2}$ Data intelligence approaches to the impact of the pandemic have since shown the full extent of the challenges that we are facing within the European cancer community. During the pandemic, it's been estimated that more than 100 million cancer screening tests were missed, potentially as many as one million Europeans may be walking around with an undiagnosed cancer, while one in two Europeans with cancer did not receive the surgery or chemotherapy that they needed in a timely fashion. ${ }^{6}$

Fear of contracting covid-19 has meant European citizens were afraid to go to their family doctor; in the UK, for example, a study found that nearly half of people who had potential cancer symptoms in the first wave of the pandemic did not go to their GP. ${ }^{7}$ It also made patients reluctant to attend screening appointments, and cancer patients less likely to go to their hospital or cancer centre for treatment. Surveys from patient advocacy organisations corroborated this finding, ${ }^{8}$ while also highlighting the sense of isolation that many patients with cancer experienced. In many countries, specialist oncology staff were redeployed to covid wards, consequently exacerbating pre-existing staff shortages and creating a backlog of care. The impact on the cancer workforce has also been profound. One survey found that as many as four out of 10 oncology professionals experienced feelings of burnout during the pandemic and, worryingly, three out of 10 exhibited signs of clinical depression. ${ }^{9}$
Data intelligence has delineated the scale of the problem, and politicians, policy makers, and commissioners of care can't delay in responding to it. Cancer services and clearing the backlog must be at the top of the health agenda. Otherwise, the current pandemic will precipitate a future cancer epidemic.

For citizens and patients, it is critical that there are no delays in accessing optimal cancer care. Cancer services are safe, and people shouldn't hesitate to visit their doctor if they have potential cancer symptoms or to keep screening and treatment appointments. For healthcare workers, both hospital leadership and line managers must look at innovative ways to support the wellbeing of the workforce, with early interventions to address symptoms of anxiety and depression.

Reliable, rapid data intelligence must be part of our armamentarium against cancer. Getting back to the previous normal will not be enough-the covid pandemic has highlighted the fragility of our cancer health services, while presentational, diagnostic, and treatment delays that have occurred have generated a significant backlog that must be tackled. Nonetheless, we have a huge opportunity here, particularly at a European level, with the ongoing implementation of the EU Cancer Mission, ${ }^{10}$ which is delineating and supporting Europe's research efforts for cancer and feeding into the patient focused Europe's Beating Cancer Plan, ${ }^{11}$ in order to deliver better outcomes for cancer patients across the region.

We need to make use of intelligence tools ${ }^{12}$ to help inform future planning for cancer care. Getting granular data on the differential effects of the pandemic on different cancers can be crucial in showing us where to focus our efforts and in developing tailored solutions. In colorectal cancer, for example, there was a significant (90\%) drop in the NHS in the number of endoscopy procedures performed in the early stages of the pandemic. ${ }^{4}$ Yet data from faecal immunochemical testing can be used to create a triaging approach that has the potential to reduce delays and make best use of scarce diagnostic resources. ${ }^{13}$ We need to reimagine how cancer services should work during this recovery period and in future, deploying robust and rapid cancer intelligence to drive a data-informed, research-empowered cancer health system that truly delivers citizen and patient centred care.

The covid-19 pandemic has placed us at a crucial inflection point in relation to cancer in Europe, but there's no time to lose in responding to this unforeseen challenge. Cancer intelligence can 


\section{OPINION}

\section{illuminate the path to take, but it must be supported by the commitment and investment of governments.}

Competing Interests: ML has received honorararia from Pfizer, EMD Serono, Roche, and Carnall Farrar unrelated to this work. ML has received an unrestricted educational grant from Pfizer unrelated to this work. MC has no competing interests to declare.

Provenance and peer review: not commissioned; not peer reviewed.

1 Lai AG, Pasea L, Banerjee A, etal. Estimated impact of the COVID-19 pandemic on cancer services and excess 1-year mortality in people with cancer and multimorbidity: near real-time data on cancer care, cancer deaths and a population-based cohort study. BMJ Open 2020;10:e043828. doi: 10.1136/bmjopen-2020-043828. pmid: 33203640

2 Dinmohamed AG, Visser O, Verhoeven RHA, etal. Fewer cancer diagnoses during the COVID-19 epidemic in the Netherlands. Lancet Oncol 2020;21:750-1. doi: 10.1016/\$1470-2045(20)30265-5. pmid: 32359403

3 Sud A, Torr B, Jones ME, etal. Effect of delays in the 2-week-wait cancer referral pathway during the COVID-19 pandemic on cancer survival in the UK: a modelling study. Lancet Oncol 2020;21:1035-44. doi: 10.1016/S1470-2045(20)30392-2. pmid: 32702311

4 Ho KMA, Banerjee A, Lawler M, Rutter MD, Lovat LB. Predicting endoscopic activity recovery in England after COVID-19: a national analysis. Lancet Gastroenterol Hepatol 2021;6:381-90. doi: 10.1016/S2468-1253(21)00058-3. pmid: 33713606

5 Lawler M. Cancer must not be the forgotten "C" in the fight against covid-19. BMJ Opinion. 11 May 2021. https://blogs.bmj.com/bmj/2021/05/11/cancer-must-not-be-the-forgotten-c-in-thefight-against-covid-19/

6 Covid-19 \& Cancer Data Intelligence. European Cancer Organisation. https://www.europeancancer.org/timetoact/impact/data-intelligence

7 Almost half of people with possible cancer symptoms didn't see GP in first wave of pandemic. Cancer Research UK. 25 February 2021. https://news.cancerresearchuk.org/2021/02/25/almosthalf-of-people-with-possible-cancer-symptoms-didnt-see-gp-in-first-wave-of-pandemic/

8 Voisin MR, Oliver K, Farrimond S, et al. Brain tumors and COVID-19: the patient and caregiver experience. Neurooncol Adv. 2020 23;2(1):vdaa104. doi: 10.1093/noajnl/vdaa104.

9 Lim KHJ, Murali K, Kamposioras K, etal. The concerns of oncology professionals during the COVID-19 pandemic: results from the ESMO Resilience Task Force survey II. ESMO Open 2021;6:100199. doi: 10.1016//.esmoop.2021.100199. pmid: 34217129

10 Conquering cancer, mission possible. European Commission. September 2020. https://ec.europa.eu/info/publications/conquering-cancer-mission-possible_en

11 Europe's Beating Cancer Plan. A new EU approach to prevention, treatment and care. European Commission. 3 February 2021. https://ec.europa.eu/commission/presscorner/detail/en/ip_21_342

12 Time To Act Data Navigator - Build Back Better. European Cancer Organisation. YouTube video. 17 November 2021. https://www.youtube.com/watch?v=gh8Uek9Bg00

13 Loveday C, Sud A, Jones M, et al. Prioritisation by FIT to mitigate the impact of delays in the 2-week wait colorectal cancer referral pathway during the COVID-19 pandemic: a UK modelling study Gut 2020 Aug 27:gutjnl-2020-321650. doi: 10.1136/gutjnl-2020-321650 